\title{
PIC Simulations of Ka-band Ultra-Short Pulse Oscillator with Resonance Cyclotron Absorber in the Feedback Loop
}

\author{
N.S. Ginzburg ${ }^{1,2}$, G.G. Denisov ${ }^{1}$, M.N. Vilkov ${ }^{1}$, I.V. Zotova ${ }^{1}$, A.S. Sergeev ${ }^{1}$, \\ S.V. Samsonov ${ }^{1}$, A. Marek ${ }^{3}$, and J. Jelonnek ${ }^{3}$ \\ ${ }^{1}$ Institute of Applied Physics RAS, Nizhny Novgorod, Russia, vilkovmn@ipfran.ru, \\ ${ }^{2}$ Nizhny Novgorod State University Nizhny Novgorod, Russia \\ ${ }^{3}$ Karlsruhe Institute of Technology, Karlsruhe, Germany
}

In $[1,2]$ it was shown that periodical trains of ultrashort microwave pulses (USP) can be produced in a two-section microwave oscillator consisting of an amplifier and a nonlinear (saturable) absorber in the feedback loop. In such a system, the process of pulse formation is based on the effect of passive modelocking which is well known in laser physics [3]. At the present moment, the Ka-band USP oscillator with a broadband helical-waveguide gyro-TWT [4] as an active unit is under development at the Institute of Applied Physics. The saturable absorber will be realized in an auxiliary section based on the cyclotron resonance interaction with an initially rectilinear magnetized electron beam. In this case the nonlinearity of absorption is provided due to relativistic dependence of a gyrofrequency on the electrons energy. The basic parameters of the USP oscillator have been defined on the basis of a simple averaged model. This paper is devoted to the full-scale particle-in-cell (PIC) simulations of such a system based on the software CST Studio Suite [5] aimed to optimize the parameters taking into account the real conditions of the planning experiment.

For simulations the computer model has been developed, in which the helical-waveguide amplifier and the cyclotron absorber were installed opposite each other (see Fig. 1). The gyro-TWT operates at the second cyclotron harmonic while the fundamental cyclotron resonance is used in the absorber unit. From the cathode ends both sections were bounded by resonance reflectors. The independent electron beams with the given parameters were entered the calculated space from the left and right ends of the corresponding sections and, after interaction, deflected in a strong transverse magnetic field to the waveguide wall. Between the oscillator sections there was a polarization-selective waveguide coupler which allows simulating the branching of the useful power to the load. Thus, the initial microwave pulse could be fed to the single-mode waveguide port 1 , from which a part of the power (from 20 to $60 \%$ ) in the form of an $\mathrm{x}$ polarized $\mathrm{TE}_{1,1}$ wave (with the $\mathrm{x}$-component of the electric field on the system axis) came in the $-\mathrm{z}$ direction to the input of the left polarizer, where it was converted into a rotating wave. This circularly polarized $\mathrm{TE}_{1,1}$ wave propagates almost without losses towards the electron beam of the amplifier, reaches the reflector at the left end of the system, where, with a coefficient of $98-100 \%$, it is reflected in the opposite $+\mathrm{z}$ direction, without changing direction of rotation. Propagating further in the section of the helicalwaveguide gyro-TWT along with the electron beam whose particles possess the required given transverse velocity, the rotating wave $\mathrm{TE}_{1,1}$ in the region with the increasing amplitude of the corrugation is adiabatically transformed into the operating mode of the helical waveguide, which is amplified by an electron beam at the second cyclotron harmonic. Further, this wave is converted at the corrugation exit back into the rotating wave $\mathrm{TE}_{1,1}$, which once again passes through the polarizer, but already in the $+\mathrm{z}$ direction. In this case, the circularly polarized wave is converted back to the linearly polarized one, but with the y-component of the electric field on the system axis. Such a mode (practically not perturbed by the coupler) transforms into a rotating wave in the right polarizer, passes without resonance interaction towards the beam in the absorbing section, reflects without changing the direction of rotation, and on the return path (in the $-\mathrm{z}$ direction) is resonantly absorbed, betraying its energy into rotational energy of electrons. Passing again through the polarizer in the $-\mathrm{z}$ direction, it becomes $\mathrm{X}$ polarized, which will result in a part of its power (from 40 to $80 \%$ ) being output through the waveguide port 2. The remainder will go to the input of the amplifier, thereby closing the feedback loop.

In accordance with the planned experiments, the frequency of $34 \mathrm{GHz}$ was selected as the operating

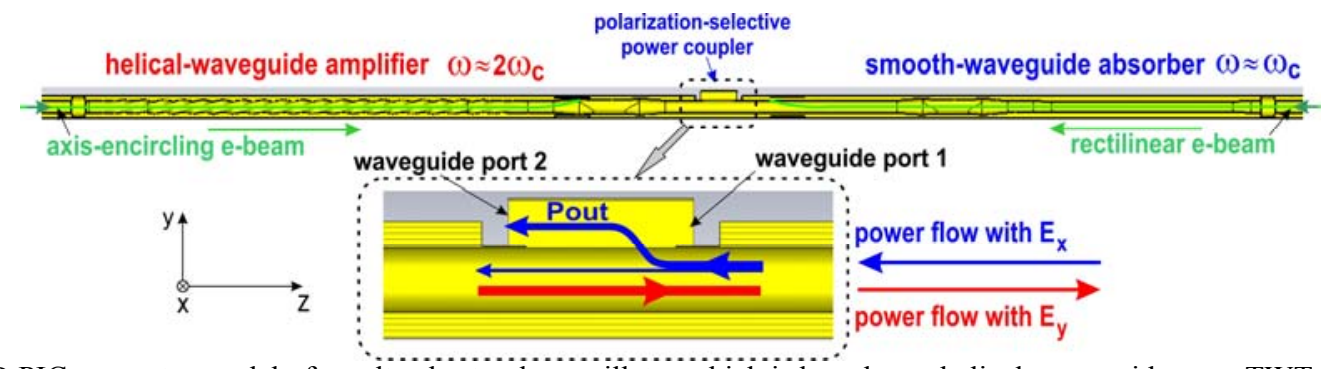

Fig. 1. 3D PIC computer model of an ultrashort pulse oscillator which is based on a helical-waveguide gyro-TWT and a resonance cyclotron absorber with a rectilinear electron beam. 
one. The gyro-TWT was fed by an encircling electron beams with the following parameters: electron energies of $70 \mathrm{keV}$, beam current of $6 \ldots 10 \mathrm{~A}$, pitch factor 1 ... 1.3, magnetic field of 0.68..0.7 T. Parameters of corrugation were chosen in such a way as to provide an optimum amplification of ultrashort pulses [2].

At the initial stage, in order to clarify the parameters of the saturable cyclotron absorber, it was simulated separately. The aim of optimization was providing the necessary nonlinearity and the frequency bandwidth of the absorption. The most optimal properties were achieved for the following parameters of an electron beam: the energy of $50 \mathrm{keV}$ and electron current of 1.2 A. The operating mode was a fundamental mode of regular round waveguide with a radius of $3 \mathrm{~mm}$ and the length of $83 \mathrm{~mm}$. The guiding magnetic field of $1.07 \mathrm{~T}$ was chosen in such a way as to provide the grazing between the electron line and operating mode dispersion characteristic. Under such conditions, local interaction (i.e. interaction with minimal slippage) of every segment of the microwave pulse with a corresponding fraction of the electron beam takes place. Such arrangement is equivalent to the requirement of minimization of the absorber relaxation time in laser physics [3]. For chosen parameters the frequency dependence of the absorption ration is presented in Fig. 2 for different levels of incident power. One can see that the system exhibits properties of a saturable absorber when a relatively weak signal ( $P_{\text {in }}=10 \mathrm{~kW}$ in Fig. 2) is attenuated significantly stronger than a signal with high power ( $P_{i n}=100 \mathrm{~kW}$ in Fig. 2). It is important that these properties are maintained over a wide frequency range.

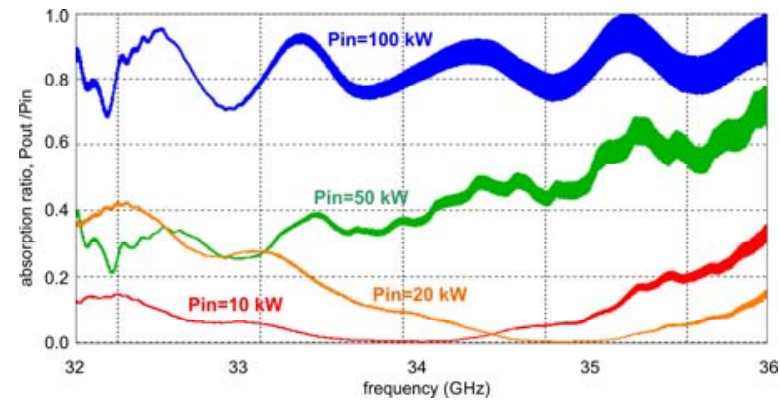

Fig. 2. Dependence of the absorption ratio of the cyclotron absorber on the frequency for various levels of incident power.

In further simulations of the complete system, the absorber and amplifier parameters are varied within certain limits in order to optimize the generation regime. The obtained results confirm the practical feasibility of the chosen concept of an USP oscillator (see Fig. 3). The highest stability of generation (pulseto-pulse reproducibility) is achieved in the hard excitation mode when generation develops after injection of an external pulse into the system for bleaching of the absorber. In this case, the pulse repetition period is $\sim 7 \mathrm{~ns}$, which roughly corresponds to the delay time in the feedback loop. In the soft excitation mode, the most regular pulse sequences are obtained with a delayed switching on the absorber with respect to the amplifying section due to a linear increase in the cur- rent (Fig. 3b). It should be noted that the coherence of pulses (the time stability of the phase of microwave oscillations) is maintained throughout the counting time (in this case $300 \mathrm{~ns}$ ), in spite of some variations in the amplitude of the generated pulses.

a)

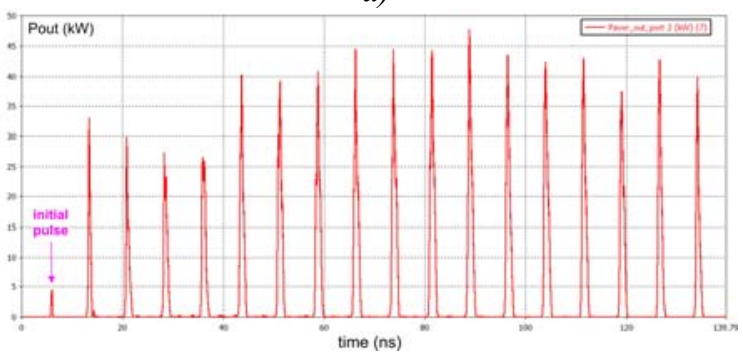

b)

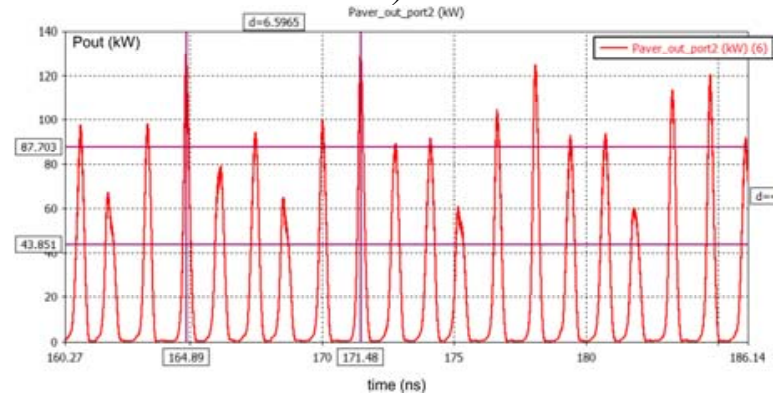

Fig. 3. Results of PIC simulations for generation of a sequence of ultrashort pulses in a two-section microwave oscillator with passive mode locking. The hard (a) and soft (b) self-excitation modes are presented.

Thus, the developed computer model, on the one hand, adequately describes the main characteristics of electron-wave interaction in the system under study, and, on the other hand, requires moderate computer resources. The simulation carried out based on this basis is a full-fledged numerical experiment that made it possible to determine the parameters for the practical realization of the ultrashort pulse generator with the passive mode-locking in microwave electronics.

This work was supported by Russian Scientific Foundation (RSCF), grant No. 16-42-01078.

\section{References}

1. Ginzburg N.S., Denisov G.G., Vilkov M.N. et al. Generation of "gigantic" ultra-short microwave pulses based on passive mode-locking effect in electron oscillators with saturable absorber in the feedback loop // Phys. Plasmas. 2016. V. 23. P. 050702-1-4.

2. Ginzburg N. S., Denisov G. G., Vilkov M. N. et al. Generation of train of ultrashort microwave pulses by two coupled helical gyro-TWTs operating in regimes of amplification and nonlinear absorption // Physics of Plasmas. 2017. V. 24. P. 023103-1-5.

3. Haus H.A. Mode-Locking of Lasers // IEEE J. of Select. Topics in Quant. Electron. 2000. V. 6, No. 6. P. 11731185.

4. Denisov G.G., Bratman V.L., Cross A.W. et al. Gyrotron Traveling Wave Amplifier with a Helical Interaction Waveguide // Phys. Rev. Lett. 1998. V. 81, No. 25. P. 56805683.

5. https://www.cst.com 